\title{
Rostral Horn Evolution Among Agamid Lizards of the Genus Ceratophora Endemic to Sri Lanka
}

James A. Schulte II $^{1}$, J. Robert Macey ${ }^{2}$, Rohan Pethiyagoda ${ }^{3}$, Allan Larson ${ }^{1}$

${ }^{1}$ Department of Biology, Box 1137, Washington University, St. Louis, Missouri 63130 (schulte@biology.wustl.edu, $\underline{\text { larson@wustlb.wustl.edu) }}$

${ }^{2}$ Department of Comparative Genomics, Joint Genome Institute and Lawrence Berkeley National Laboratory, 2800 Mitchell Drive, Walnut Creek, CA 94598-1631

(jrmacey@1bl.gov)

${ }^{3}$ Wildlife Heritage Trust, 95 Cotta Road, Colombo 8, Sri Lanka (rohan@wht.org)

*To whom correspondence should be addressed: Phone (314) 935-5302, FAX (314) 9354432, E-mail: schulte@ biology.wustl.edu

Running title: Horn Evolution in Agamid Lizards

Key Words: Reptilia, Squamata, Iguania, Agamidae*, Ceratophora, Mitochondrial DNA, Phylogenetics, Character Evolution. 
The first phylogenetic hypothesis for the Sri Lankan agamid lizard genus Ceratophora is presented based on 1670 aligned base positions (472 parsimony informative) of mitochondrial DNA sequences, representing coding regions for eight tRNAs, ND2, and portions of ND1 and COI. Phylogenetic analysis reveals multiple origins and possibly losses of rostral horns in the evolutionary history of Ceratophora. Our data suggest a middle Miocene origin of Ceratophora with the most recent branching of recognized species occurring at the Pliocene/Pleistocene boundary. Haplotype divergence suggests that an outgroup species, Lyriocephalus scutatus, dates at least to the Pliocene. These phylogenetic results provide a framework for comparative studies of the behavioral ecological importance of horn evolution in this group. 


\section{INTRODUCTION}

Squamate reptiles exhibit substantial variation in body ornamentation, including crests, frills, spines, casques, dewlaps, and horns. Rostral protuberences or "horns" are rare and all occurrences are in Iguania. Within the iguanian family Iguanidae, horns are confined to three species of Anolis from the Amazonian Andes (Williams, 1979). Within Acrodonta, horns occur only in family Chamaeleonidae and the agamid subfamily, Draconinae (Macey et al., 2000b). Phylogenetic analysis of Chamaelenonidae suggests two evolutionary origins and three losses of horns in this group (Townsend and Larson, 2001).

The agamid lizard genus Ceratophora contains five species endemic to Sri Lanka, most exhibiting modifications of the rostrum in the form of a rhinocerus-like horn (see color plates in Pethiyagoda and Manamendra-Arachchi, 1998). Horn morphology is radically different for each species (Table 1). Only two other monotypic genera within Agamidae* (asterisk denotes a metataxon, whose monophyly is uncertain) contain a prominent, horn-like rostral appendage: Harpesaurus, which has a forked appendage, and Thaumatorhynchus, which has a single, cylindrical horn (Manthey and Schuster, 1996).

As originally described, Ceratophora contained three species, C. aspera, $C$. stoddartii, and C. tennentii, each exhibiting prominent rostral appendages. Ceratophora aspera, a ground-dwelling species, and the semi-arboreal $C$. stoddartii occupy the largest geographic area of the described species, whereas the arboreal species, $C$. tennentii, is found only in the Knuckles mountain range. Recently, Pethiyagoda and ManamendraArachchi (1998) reviewed the taxonomic content of Ceratophora and described two additional species, C. erdeleni and C. karu. The semi-arboreal C. erdeleni has a rudimentary or nonexistent rostral appendage and is otherwise very similar morphologically to $C$. stoddartii, whereas $C$. karu is ground-dwelling and the only fast-moving lizard containing a rostrum composed of numerous pointed scales. The rostrum of $C$. karu lacks the fleshy protuberence characteristic of horned Ceratophora, however. Both of these 
species have restricted distributional ranges in the Morningside Forest Reserve of southeastern Sri Lanka and surrounding areas. Species of Ceratophora are allopatric to each other, except $C$. erdeleni and $C$. karu, whose ranges overlap in the Morningside Forest Reserve.

Phylogenetic relationships of all species of Ceratophora are examined using 1741 aligned positions (472 informative) of mitochondrial DNA sequence. The region sequenced encodes part of ND1 (NADH dehydrogenase subunit 1), tRNA ${ }^{\text {Gln }}, \mathrm{tRNA}^{\text {Ile }}$, $\mathrm{tRNA}^{\mathrm{Met}}$, ND2 (NADH dehydrogenase subunit 2), $\mathrm{tRNA}^{\mathrm{Trp}}, \mathrm{tRNA}^{\mathrm{Ala}}, \mathrm{tRNA}^{\mathrm{Asn}}$, replication origin for the light strand $\left(\mathrm{O}_{\mathrm{L}}\right)$, tRNA ${ }^{\mathrm{Cys}}$, $\mathrm{tRNA}^{\mathrm{Tyr}}$, and part of COI (cytochrome oxidase I). The previously published sequence of $C$. aspera (Macey et al., 2000b) is compared to a new sequence from the same population. Three populations of $C$. stoddartii are examined to assess phylogenetic divergence within this wide-ranging species. Phylogenetic inferences are used to generate hypotheses of horn evolution within

\section{Ceratophora.}

A recent revision of Agamidae* by Macey et al. (2000b) placed Ceratophora within subfamily Draconinae. In their analysis, Lyriocephalus scutatus and Cophotis ceylonica formed a clade strongly supported as the sister group to Ceratophora. Sequences from Cophotis and Lyriocephalus, including an additional population of Lyriocephalus scutatus, are used here as closest outgroups. In addition, two other southeast Asian members of Draconinae, Japalura flaviceps and Aphaniotis fusca, are selected as outgroups based on the phylogenetic hypothesis of Macey et al. (2000b). All four of these sequences are reported in Macey et al. (2000b). Sequences for Ceratophora karu, C. tennentii, and $C$. erdeleni that were previously reported for approximately 300 base pairs (Macey et al., 2000c) have been extended for the full 1741 base-pair region analyzed here. 


\section{MATERIALS AND METHODS}

\section{Specimen Information}

See Table 1 for museum numbers, localities of voucher specimens from which DNA was extracted, and GenBank accession numbers for DNA sequences. Complete locality data are deposited in GenBank files.

\section{Laboratory Protocols}

Genomic DNA was extracted from liver or muscle using Qiagen QIAamp tissue kits. Amplification of genomic DNA was conducted using a denaturation at $94^{\circ} \mathrm{C}$ for 35 sec, annealing at $50^{\circ} \mathrm{C}$ for $35 \mathrm{sec}$, and extension at $70^{\circ} \mathrm{C}$ for $150 \mathrm{sec}$ with $4 \mathrm{sec}$ added to the extension per cycle, for 30 cycles. Negative controls were run on all amplifications to check for contamination. Amplified products were purified on $2.5 \%$ Nusieve GTG agarose gels and reamplified under the conditions described above. Reamplified doublestranded products were purified on $2.5 \%$ acrylamide gels (Maniatis et al., 1982). Template DNA was eluted from acrylamide passively over three days with Maniatis elution buffer (Maniatis et al., 1982). Cycle-sequencing reactions were run using the Promega fmol DNA sequencing system with a denaturation at $95^{\circ} \mathrm{C}$ for $35 \mathrm{sec}$, annealing at $45-60^{\circ} \mathrm{C}$ for $35 \mathrm{sec}$, and extension at $70^{\circ} \mathrm{C}$ for $1 \mathrm{~min}$ for 30 cycles. Sequencing reactions were run on Long Ranger sequencing gels for 5-12 hours at $38-40^{\circ} \mathrm{C}$.

Two primer pairs were used to amplify genomic DNA from the ND1 gene to the COI gene: L3914 and H4980, and L4437 and H5934. Both strands were sequenced using L3914, L4178b, L4160, L4437, L4831, L4882b, H5617b, L5638b, and H5934. Primers L4831, L4178b, L4831, H4980, L5638b, and H5934 are from Macey et al. (1997a). L3914 is from Macey et al. (1998b) which is erroneously listed there as L3878. L4160 is from Kumazawa and Nishida (1993). L4437 and H5617b are from Macey et al. (1997b). L4882b is from Macey et al. (2000b). Primer numbers refer to the 3' end on the human 
mitochondrial genome (Anderson et al., 1981), where L and H denote extension of light and heavy strands, respectively.

\section{Phylogenetic Analysis}

DNA sequences were aligned manually. Positions encoding part of ND1, all of ND2, and part of COI were translated to amino acids using MacClade (Maddison and Maddison, 1992) for confirmation of alignment. Alignment of sequences encoding tRNAs was based on secondary structural models (Kumazawa and Nishida, 1993; Macey and Verma, 1997). Secondary structures of tRNAs were inferred from primary structures of the corresponding tRNA genes using these models. Unalignable regions were excluded from phylogenetic analyses (see Results).

Phylogenetic trees were estimated using PAUP* beta version 4.0b4a (Swofford, 2000) with branch-and-bound searches. Bootstrap resampling (Felsenstein, 1985a) was applied to assess support for individual nodes using 1000 bootstrap replicates with branchand-bound searches. Decay indices (= "branch support" of Bremer, 1994) were calculated for all internal branches of the tree using branch-and-bound searches that retained suboptimal trees. We obtained the decay index by tabulating the minimum increase in number of steps resulting from removal of the node of interest from the overall shortest tree.

Evolution of rostral horns among Ceratophora species was reconstructed using MacClade (Maddison and Maddison, 1992) on the single overall most parsimonious tree. The outgroup species, Aphaniotis fusca, Japalura flaviceps, and Cophotis ceylonica do not contain a rostral protuberance; however, the outgroup species, Lyriocephalus scutatus, does contain a knob-like rostral structure.

Wilcoxon signed-ranks tests (Templeton, 1983; Felsenstein, 1985b) were used to examine statistical significance of the shortest tree relative to alternative hypotheses. All 
tests were conducted as two-tailed tests (Felsenstein, 1985b). Tests were performed using PAUP* (Swofford, 2000), which incorporates a correction for tied ranks.

Alternative phylogenetic hypotheses were tested using the most parsimonious phylogenetic topologies compatible with them. To find the most parsimonious tree(s) compatible with a particular phylogenetic hypothesis, phylogenetic topologies were constructed using MacClade (Maddison and Maddison, 1992) and analyzed as constraints using PAUP* (Swofford, 2000) with branch-and-bound searches.

\section{RESULTS}

\section{Assessment of Homology and Sequence Alignment}

Protein-coding genes are alignable except the C-terminal end of ND1, which is excluded because of questionable alignment (positions 82-86). Gaps are placed in the ND2 gene sequence at nucleotide positions 1252-1254 in the outgroup, Japalura flaviceps.

Among tRNA genes, several loop regions are unalignable as are noncoding regions between genes. The dihydrouridine (D) loops for genes encoding tRNA ${ }^{\text {Ile }}$ (positions 176182) and tRNA ${ }^{\operatorname{Trp}}$ (positions 1345-1354) are excluded from analyses. Part of the variable loop for the gene encoding tRNA ${ }^{\text {Asn }}$ (positions 1516-1518) is not used. The loop of the origin for light-strand replication $\left(\mathrm{OL}_{\mathrm{L}}\right.$, positions 1563-1585) between the $\mathrm{tRNA}^{\mathrm{Asn}}$ and tRNA $^{\mathrm{Cys}}$ genes is not alignable and therefore not used for phylogenetic analysis. The $\mathrm{T} \psi \mathrm{C}(\mathrm{T})$ loop, and part of the T-stem for the gene encoding tRNA ${ }^{\mathrm{Cys}}$ (positions 15971605) and the T-loop for the gene encoding tRNA ${ }^{\mathrm{Tyr}}$ (positions 1656-1659) are excluded from analyses. Noncoding sequences between the tRNA ${ }^{\text {Ala }}$ and tRNA ${ }^{\text {Asn }}$ genes (positions 1479-1488) are not used. Excluded regions comprise 4\% of aligned sequence positions (71 of 1741 positions). 
Several observations suggest that DNA sequences reported are from the mitochondrial genome and not nuclear-integrated copies of mitochondrial genes (see Zhang and Hewitt, 1996). Protein-coding genes do not contain premature stop codons, and sequences of tRNA genes appear to code for tRNAs with stable secondary structures, indicating functional genes. In addition, all sequences show strong strand bias against guanine on the light strand $(\mathrm{G}=11.4-13.4 \%, \mathrm{~A}=32.6-36.7 \%, \mathrm{~T}=21.9-23.7 \%$, and $\mathrm{C}=28.6-32.0 \%$ ), which is characteristic of the mitochondrial genome but not the nuclear genome (Macey et al., 1997a; McGuire and Heang, 2001).

\section{Genic Variation}

Variation in phylogenetically informative positions (parsimony criterion) is observed among all tRNA and protein-coding genes (Table 2). Phylogenetically informative sites are predominately from protein-coding regions ( $83 \%$ of informative sites) with most of the variation observed in third codon positions (48\%). However, first and second codon positions, as well as stem and non-stem regions of tRNA genes, together contributed over half of the phylogenetically informative sites $(21 \%, 13 \%, 13 \%, 4 \%$, respectively). Therefore, no single set of characters dominates the phylogenetic analysis.

\section{Phylogenetic Relationships}

A single overall most parsimonious tree is produced from analysis of 12 aligned DNA sequences containing 1670 base positions, of which 472 are phylogenetically informative (Fig. 1, Table 2). Phylogenetic relationships are well resolved, receiving bootstrap values above $95 \%$ and decay indices greater than 5 for all nodes except two. A clade comprising Lyriocephalus, Cophotis, and Ceratophora is well supported (bootstrap 100\%, decay index 42). The sister-taxon relationship of Lyriocephalus and Cophotis is well-supported (bootstrap 99\%, decay index 17). Monophyly of Ceratophora is strongly supported by a bootstrap value of $97 \%$ and a decay index of 13 . The small, ground- 
dwelling species, $C$. aspera, is the sister taxon to a moderately supported group containing the remaining species (bootstrap 76\%, decay index 4), and the two C. aspera sequences from the same population are identical. The small, ground-dwelling, $C$. karu, is the sister taxon to a group containing $C$. tennentii, $C$. erdeleni, and all populations of $C$. stoddartii (bootstrap 100\%, decay index 17). The large-bodied, semi-arboreal species, C. erdeleni and C. stoddartii, form a well supported, monophyletic group (bootstrap 100\%, decay index 48). The three populations of $C$. stoddartii form a moderately supported monophyletic group to the exclusion of the morphologically similar C. erdeleni (bootstrap 86\%, decay index 4). Within C. stoddartii, populations from Tangamalai and Namunukula are strongly supported as a group (bootstrap 96\%, decay index 5).

We investigate whether presence of horns in Ceratophora arose once or multiple times. When occurrence of horns is mapped onto the overall shortest tree using MacClade (Fig. 2), multiple origins or losses of horns are depicted on the overall shortest tree (Fig. 1). To test the hypothesis of multiple evolutionary events involving horns, the Wilcoxon signed-ranks test is applied. When the overall shortest tree from analysis of the DNA sequence data is compared to the shortest alternative tree ( $\mathrm{A}$ in appendix 1 ) in which all species containing prominent horns form a monophyletic group, this alternative is rejected in favor of the overall shortest tree $\left(n=122, T_{s}=5.98, p<0.0001 * *\right)$. When the overall shortest tree from analysis of the DNA sequence data is compared to the shortest alternative tree (B in appendix 1) in which the two species lacking prominent horns form a clade, this alternative is rejected in favor of the overall shortest tree $\left(n=98, T_{s}=6.87, p<0.0001^{* *}\right)$. These results indicate that horns either arose multiple times or were lost multiple times among the five species of Ceratophora.

\section{DISCUSSION}

\section{Horn evolution in Ceratophora}


This study provides the first phylogenetic hypothesis for the agamid horned-lizard genus Ceratophora and reveals multiple evolutionary events involving horns among the five species. In the parsimony reconstruction (Fig. 2), horns have evolved either three times in C. aspera, $C$. tennentii, and $C$. stoddartii, or once at the base of the Ceratophora tree, followed by separate losses in $C$. karu and C. erdeleni. This latter hypothesis appears less likely given the radically different morphologies of horns among Ceratophora species (Pethiyagoda and Manamendra-Arachchi, 1998). If horns have a single evolutionary origin, horn morphology should be similar among all horned species.

Unfortunately, relatively little is known about the function of horns in Ceratophora. Two primary hypotheses have been proposed for the function of ornamentation in iguanian lizards. One hypothesis is that horns are important for species recognition (Rand, 1961). This hypothesis was suggested because closely related species of African chameleons have very different ornamentation types when occurring in sympatry. This hypothesis is unlikely for Ceratophora, because the only two sympatric species in this genus do not have horns.

Another hypothesis is that horns function in intraspecific communication, primarily during male-male interactions. The agamid frillneck lizard of Australia, Chlamydosaurus kingii, displays more elaborate head-bobs, push-ups, mouth-opening, and full erection of the frill during male-male interactions (Shine, 1990). However, partial erection of the frill is observed during male-female interactions and possible predator interactions. Watkins (1998) demonstrates that the crest in males of the iguanid lizard, Microlophus occipitalis, is relatively larger, and is elevated only during interactions between males, never during malefemale encounters. This latter hypothesis is the most plausible explanation for horns in Ceratophora. In general, horns of male Ceratophora are larger than female horns and are movable (Ilangakoon, 1990; Pethiyagoda and Manamendra-Arachchi, 1998). More detailed ecological work is necessary to determine function of horns of Ceratophora. 


\section{Dating Horn Evolution and Cladogenesis in Ceratophora}

Rates of molecular evolution for this of mitochondrial DNA segment are reported in the agamid lizard genus Laudakia (Macey et al., 1998a, 2000a) as $0.65 \%$ change per million years per lineage. This approximate calibration has been corroborated for fishes (0.65\%), bufonid frogs (0.69\%), and gekkonid lizards (0.57\%) (Bermingham et al., 1997; Macey et al., 1998a, 2000a). After 10 million years, mitochondrial DNA is expected to saturate (Moritz et al., 1987); therefore, a linear relationship of nucleotide substitutions and time is not anticipated. If this rate of molecular evolution is approximately correct for other agamid lizards, then divergence times in the subfamily Draconinae and within Ceratophora are expected to exceed 23 million years and 13 million years, respectively (Table 3). All branching events among the endemic Sri Lankan genera, Ceratophora, Lyriocephalus, and Cophotis are at least 14 million years ( 18\% sequence divergence; Fig. 1; Table 3).

Our data suggest a Miocene origin of Ceratophora with the most recent branching of recognized species occurring at the Pliocene/Pleistocene boundary. The first divergence among Ceratophora lineages is estimated to be approximately 12.6 MYA ( 16\% sequence divergence; Table 3) between $C$. aspera and the common ancestor of the remaining four species. Two million years later at $\sim 10.8$ MYA (Fig. 1; Table 3) the lineage leading to $C$. karu, without a prominent horn, split from the lineage ancestral to the remaining species. Ceratophora tennentii separated at $~ 7.1$ MYA (Fig. 1; Table 3) from a lineage giving rise to C. erdeleni and C. stoddartii. At approximately 2.4 MYA, (Fig. 1; Table 3) divergence occurred between ancestral lineages of $C$. erdeleni, which lacks prominent horns, and $C$. stoddartii, which has a unique, singly-scaled, pointed horn. Divergence of the two populations of Lyriocephalus, between which relatively little morphological divergence has occurred, is approximately 4.3 MYA (Fig. 1; Table 3).

The large morphological differences observed among horn structures of C. aspera, C. stoddartii, and C. tennentii, and the fact that these species do not form a clade, suggest three separate origins of horns in Ceratophora. Our results suggest that horns in C. aspera 
and $C$. tennentii arose no earlier than middle Miocene, and that horns of $C$. stoddartii arose no earlier than late Pliocene. More detailed studies of relationships among populations within Ceratophora species and their ecological attributes are needed to generate more detailed hypotheses regarding horn evolution in this group.

\section{APPENDIX 1}

\section{Trees Used in Wilcoxon signed-ranks Tests}

The shortest alternative trees recovered from phylogenetic analyses imposing constraints are listed with tree lengths in parentheses.

A. The most parsimonious tree depicting the alternative hypothesis that Ceratophora with prominent horns form a monophyletic group (1579 steps): (Aphaniotis fusca, (Japalura flaviceps, ((Cophotis ceylanica, (Lyriocephalus scutatus1, L. scutatus2)), ((((Ceratophora aspera, C. tennentii), (C. stoddartiil, (C. stoddartii2, C. stoddartii3))), C. erdeleni), C. karu)))).

B. The most parsimonious tree depicting the alternative hypothesis that Ceratophora without prominent horns form a monophyletic group (1581 steps): (Aphaniotis fusca, (Japalura flaviceps, ((Cophotis ceylanica, (Lyriocephalus scutatus 1, L. scutatus2)), (Ceratophora aspera, (((C. karu, C. erdeleni), (C. stoddartiil, (C. stoddartii2, C. stoddartii3))), C. tennentii))))).

\section{ACKNOWLEDGMENTS}

This work was supported by grants from the National Science Foundation (predoctoral fellowship to J.A.S.; DEB-9726064 to A.L., J.R.M and Theodore J. Papenfuss; DEB-9318642 and DEB-9982736 to J.B. Losos, K. de Queiroz, and A.L.). This work is LBNL-48056 and was partially performed under the auspices of the U.S. Department of Energy by the University of California, Lawrence Berkeley National Laboratory under contract No. DE-AC03-76SF00098. 


\section{REFERENCES}

Anderson, S., Bankier, A. T., Barrell, B. G., de Bruijn, M. H. L., Coulson, A. R., Drouin, J., Eperon, I. C., Nierlich, D. P., Roe, B. A., Sanger, F., Schreier, P. H., Smith, A. J. H., Staden, R., and Young, I. G. (1981). Sequence and organization of the human mitochondrial genome. Nature 290:457-465.

Bermingham, E., McCafferty, S. S., and Martin, A. P. (1997). Fish biogeography and molecular clocks: Perspectives from the Panamanian Isthmus. In "Molecular Systematics of Fishes" (T. D. Kocher and C. A. Stepien, Eds.), pp. 113-128, Academic Press, San Diego.

Bremer, K. (1994). Branch support and tree stability. Cladistics 10:295-304.

Felsenstein, J. (1985a). Confidence limits on phylogenies: an approach using the bootstrap. Evolution 39: 783-791.

Felsenstein, J. (1985b). Confidence limits on phylogenies with a molecular clock. Syst. Zool. 34:152-161.

Ilangakoon, A. (1990). Observations on the horned lizard, Ceratophora stoddartii. Loris 19:44-45.

Kumazawa, Y., and Nishida, M. (1993). Sequence evolution of mitochondrial tRNA genes and deep-branch animal phylogenetics. J. Mol. Evol. 37:380-398.

Macey, J. R., Larson, A., Ananjeva, N. B., Fang, Z., and Papenfuss, T. J. (1997a). Two novel gene orders and the role of light-strand replication in rearrangement of the vertebrate mitochondrial genome. Mol. Biol. Evol. 14:91-104.

Macey, J. R., Larson, A., Ananjeva, N. B., and Papenfuss, T. J. (1997b). Evolutionary shifts in three major structural features of the mitochondrial genome among iguanian lizards. J. Mol. Evol. 44:660-674.

Macey, J. R., Schulte II, J. A., Ananjeva, N. B., Rastegar-Pouyani, N., Larson, A., and Papenfuss, T. J. (1998a). Phylogenetic relationships among agamid lizards of the 
Laudakia caucasia species group: Testing hypotheses of biogeographic fragmentation and an area cladogram for the Iranian Plateau. Mol. Phylogenet. Evol. 10:118-131.

Macey, J. R., Schulte II, J. A., Larson, A., Fang, Z., Wang, Y., Tuniyev, B. S., and Papenfuss, T. J. (1998b). Phylogenetic relationships of toads in the Bufo bufo species group from the eastern escarpment of the Tibetan Plateau: A case of vicariance and dispersal. Mol. Phylogenet. Evol. 9:80-87.

Macey, J. R., Schulte II, J. A., Kami, H. G., Ananjeva, N. B., Larson, A., and Papenfuss, T. J. (2000a). Testing hypotheses of vicariance in the agamid lizard Laudakia caucasia from mountain ranges on the Northern Iranian Plateau. Mol. Phylogenet. Evol. 14:479-483.

Macey, J. R., Schulte II, J. A., Larson, A., Ananjeva, N. B., Wang, Y., Pethiyagoda, R., Rastegar-Pouyani, N., and Papenfuss, T. J. (2000b). Evaluating trans-Tethys migration: An example using acrodont lizard phylogenetics. Syst. Biol. 49:233256.

Macey, J. R., Schulte II, J. A., and Larson, A. (2000c). Evolution and information content of mitochondrial genomic structural features illustrated with acrodont lizards. Syst. Biol. 49:257-277.

Macey, J. R., and Verma, A. (1997). Homology in phylogenetic analysis: Alignment of transfer RNA genes and the phylogenetic position of snakes. Mol. Phylogenet. Evol. 7:272-279.

Maddison, W. P., and Maddison, D. R. (1992). "MacClade, analysis of phylogeny and character evolution, version 3.0," Sinauer, Sunderland, Mass.

Maniatis, T., Fritsch, E. F., and Sambrook, J. (1982). "Molecular Cloning: A Laboratory Manual," Cold Spring Harbor Laboratory Press, Cold Spring Harbor, New York. 
Manthey, U., and Schuster, N. (1996). “Agamid lizards,” T.F.H. Publications, Inc, Neptune City, New Jersey.

McGuire, J. A., and Heang, K. B. (2001). Phylogenetic systematics of southeast Asian flying lizards (Iguania: Agamidae: Draco) as inferred from mitochondrial DNA sequences. Biol. J. Linn. Soc. 72: 203-229.

Moritz, C., Dowling, T. E., and Brown, W. M. (1987). Evolution of animal mitochondrial DNA: relevance for population biology and systematics. Ann. Rev. Ecol. Syst. 18:269-292.

Pethiyagoda, R., and Manamendra-Arachchi, K. (1998). A revision of the endemic Sri Lankan agamid lizard genus Ceratophora Gray, 1835, with description of two new species. J. South Asian Nat. Hist. 3:1-50.

Rand, S. (1961). A suggested function of the ornamentation of East African chameleons. Copeia 1961:411-414.

Shine, R. (1990). Function and evolution of the frill of the frillneck lizard, Chlamydosaurus kingii (Sauria: Agamidae). Biol. J. Linn. Soc. 40:11-20.

Swofford, D. L. (2000). "PAUP*. Phylogenetic Analysis Using Parsimony (*and Other Methods), v. 4.0b4a," Sinauer, Sunderland, MA.

Templeton, A. R. (1983). Phylogenetic inference from restriction endonuclease cleavage site maps with particular reference to the evolution of humans and the apes. Evolution 37:221-244.

Townsend, T., and Larson, A. (2001). Molecular phylogenetics and mitochondrial genomic evolution in the Chamaeleonidae. Mol. Phylogenet. Evol. (in review).

Watkins, G. G. (1998). Function of a secondary sexual ornament: the crest in the South American iguanian lizard Microlophus occipitalis (Peters, Tropiduridae). Herpetologica 54:161-169.

Williams, E. E. (1979). South American anoles: the species groups. 2. The proboscis anoles (Anolis leavis group). Breviora 449:1-18. 
Yang, Z. (1994). Estimating the pattern of nucleotide substitution. J. Mol. Evol. 39: 306314.

Zhang, D-X., and Hewitt, G. M. (1996). Nuclear integrations: challenges for mitochondrial DNA markers. Trends Ecol. Evol. 11:247-251. 


\section{FIGURE CAPTIONS}

Figure 1. Phylogenetic relationships among Ceratophora species. Single most parsimonious tree obtained from analysis of 1670 aligned base positions (472

phylogenetically informative) of mitochondrial DNA (length $=1513$ steps, consistency index 0.716). Bootstrap values are presented above branches and decay indices are shown in bold below branches. Times of divergence for each node are denoted with arrows at that particular node (mya represents million years ago) based on the pairwise sequence divergence rate of 1.3\% per million years (Macey et al., 1998a).

Figure 2. Parsimony reconstruction of the presence of horns in the agamid lizard genus Ceratophora. The overall shortest tree as shown in figure 1 is presented. Light branches represent lineages that do not contain a prominent extension of the rostrum, dark branches are those lineages with a rostral protuberence, and hatched branches are lineages equivocal for the presence of rostral structures. Based upon large morphological differences among horns of different species, we favor four separate origins of horns in $C$. aspera, $C$. stoddartii, C. tennentii, and L. scutatus. 